pain on right side. Blood exams showed rise of CRP $(19 \mathrm{mg} / \mathrm{L})$ and normal WBC. An ultrasound performed in the A\&E department was normal. Because of the persistence of pain, we repeated an ultrasound the day after that showed an anechogenic uniloculated $18 \mathrm{~mm}$ cyst at the level of the right ovary. The report was of functional follicular cyst.

After two days of observation and worsening of the symptoms, laparoscopy was performed: a necrosis of the fimbriae due to torsion at the right fallopian tube infundibulum was found. The ischaemic fimbriae were removed according to Kroener fimbriectomy. Hystological analysis described fimbriae characterized by oedema and vascular congestion.

Discussion Torsion of infundibulum could mimic a functional annessial cyst, with a normal echogenic appearance, while a torsion of the whole Fallopian tube can be more easily detected and suspected, avoiding delayed treatment. In our case, to base the management on clinical symptoms was mandatory.

Conclusions Isolated torsion of infundibulum of Fallopian tube is a challenging diagnosis. It should be considered in case of acute lower abdominal pain in young girls. Early laparoscopy seems to be the best approach for diagnosis and treatment.

\section{OSTEOLYTIC LESIONS OF THE CALCANEUS IN PAEDIATRIC PATIENTS: RESULTS FROM A MULTICENTER STUDY}

doi:10.1136/archdischild-2012-302724.0464

${ }^{1} \mathrm{~A}$ Frings, ${ }^{2} \mathrm{D}$ Andreou, ${ }^{3} \mathrm{~F}$ Machacek, ${ }^{4} \mathrm{~B}$ LieglAtzwanger, ${ }^{5} \mathrm{M}$ Zacherl, ${ }^{6} \mathrm{R}$ Windhager, ${ }^{5} \mathrm{~A}$ Leithner. 'Medical University of Graz, Graz, Austria; ${ }^{2}$ HELIOS Klinikum Berlin-Buch, Berlin, Germany; ${ }^{3}$ Orthopaedic Hospital Gersthof, Vienna; ${ }^{4}$ nstitut of Pathology, Medical University of Graz; ${ }^{5}$ Department of Orthopaedics, Medical University of Graz, Graz; ${ }^{6}$ Department of Orthopaedics, Medical University of Vienna, Vienna, Austria

Tumours of the calcaneus are exceedingly rare. X-rays are the standard examination tool and therefore we wanted to evaluate if $\mathrm{X}$-rays alone were sufficient for proper diagnosis. Diard's classification was applied to define whether different types of lesions were characteristically distributed in the bone and in addition we analyzed whether type and/or duration of symptoms were possible indicators of malignancy.

Sixteen patients (12 male, 4 female; mean age 15 years, range 8 to 20) were identified. The definitive diagnosis was simple bone cyst $(n=10)$, aneurysmatic bone cyst $(n=3)$, intraosseous lipoma $(n=1)$ and Ewing's Sarcoma $(n=2)$. Parameters analyzed were type and duration of symptoms, tentative diagnosis, operative procedure, recurrence rate, revision and localization of the lesion according to Diard.

The main current complain was of pain without prior history of trauma in twelve cases. Symptoms preceded the definitive diagnosis for a mean time of seven (range 1 to 26) months. Discrepancies of the radiological and definitive diagnosis occurred in four of 16 cases. Applying Diard's system trabecular area number 6 was affected in 14 of 16 cases. We did not observe local recurrence for benign lesions.

In each case of an osteolytic lesion of the calcaneus a malignant tumor must be ruled out and thus, plain X-rays in two planes alone are not sufficient and should therefore be followed by MRI. Applying the Diard system different types of lesions are not characteristically distributed in the bone. Increasing pain without previous trauma should always justify further examinations.

\section{PREMENSTRUAL SYNDROME IN ADOLESCENTS OF ANAND - CROSS-SECTIONAL STUDY FROM INDIA USING PREMENSTRUAL SYMPTOMS SCREENING TOOL FOR ADOLESCENTS (PSST-A)}

doi:10.1136/archdischild-2012-302724.0465
'SV Kamat, 'AS Nimbalkar, ${ }^{2,3}$ SM Nimbalkar. 'Department of Physiology; ${ }^{2}$ Department of Pediatrics, Pramukhswami Medical College; ${ }^{3}$ Central Research Services, Charutar Arogya Mandal, Anand, India

Background and Aims Premenstrual syndrome (PMS) disrupts lives of girls who otherwise would lead more productive lives. We assessed prevalence and severity of PMS in adolescents of Anand, a small town in western India and identified probable associations with age, locality, food habits, obesity, stress, genetic influence, menorrhagia and dysmenorrhoea.

Methods Cross sectional observational study in which Premenstrual Symptoms Screening Tool for Adolescents (PSST-A) questionnaire was self-administered to screen PMS and Premenstrual Dysphoric Disorder (PMDD). 1355 girls of Gujarati ethnicity in age group 10 to 23 years with regular menstrual cycles participated. Obesity was assessed through Body Mass Index. Results were examined for associations that could indicate effect of lifestyle on PMS/PMDD.

Results The prevalence of moderate to severe PMS was $17.3 \%$ and PMDD was 4.7\%. 95.0\% girls had at least one PMS symptom, $68.8 \%$ had at least one moderate to severe PMS symptom, $49.9 \%$ had one or more physical symptoms and $89.8 \%$ had more than one PMS symptom. $37.1 \%$ had disruption of daily activities while $24.2 \%$ remained absent from school/college due to PMS. $75.4 \%$ girls felt that PMS was a normal part of menstruation and $50.0 \%$ reported moderate to severe stress. $21.4 \%$ had physical symptoms every menstrual cycle. Age, weight, BMI, stress level, dysmenorrhoea and menorrhagia contribute significantly to PMS, whereas menorrhagia, stress level and junk food contribute significantly to PMDD.

Conclusion Prevalence of moderate to severe PMS and PMDD in this population is higher than reported elsewhere. PMS interferes with day to day life significantly.

\section{SOCIAL INEQUALITIES IN ADOLESCENT HEALTH}

doi:10.1136/archdischild-2012-302724.0466

1.2PF Freitas, ${ }^{3} \mathrm{MP}$ Domingues. ${ }^{1}$ Núcleo de Orientação em Epidemiologia, Universidade do Sul de Santa Catarina UNISUL, Tubarão; ${ }^{2}$ Serviço de Saúde Pública, Universidade Federal de Santa Catarina; ${ }^{3}$ Maternidade Carmela Dutra, Florianópolis, Brazil

Objectives To investigate how socioeconomic indicators and individual variables from woman, pregnancy and delivery, can interact to influence rates of adolescent pregnancy in South Brazil.

Methods Information from the Live Birth National Information System (SINASC), concerning 419,272 deliveries taking place in the State of Santa Catarina, South Brazil, from 2003 to 2007 were analised. The study combined ecological and cross-sectional methods of data collection and analsysis. Correlations between socioeconomic indicators and rates of adolescent pregnancy were obtained. Annual rates and crude and adjusted rates, using Cox Regression, were calculated for literacy, ethnicity, duration of pregnancy and frequency to antenatal care consultations.

Results Rates of adolescent pregnancy for the 293 states of Santa Catarina in the study period was $18.26 \%$ decreasing linearly from 2003 to 2007, as a result of a decreasing in rates among women from the higher socioeconomic strata. Rates showed to be inversely associated to socioeconomic and demographic indicators. Negative correlations were found for the Human Development Index, Educational Development Index, literacy, and income Rate. The categories with higher proportions of adolescent mothers were low level of literacy, lower frequency to antenatal care, lower gestational age and non-white ethnicity. For extremes in the period the Prevalence Ratios increased for low literacy, lower frequency to antenatal care, and for non-white women.

Conclusions Adolescent pregnancy in Brazil is an important Public Health problem with socioeconomic determinants, whose effects will impact mainly those women living in the less privileged groups . 